\title{
A survey-based study identifies common but unrecognized symptoms in a large series of juvenile Huntington's disease
}

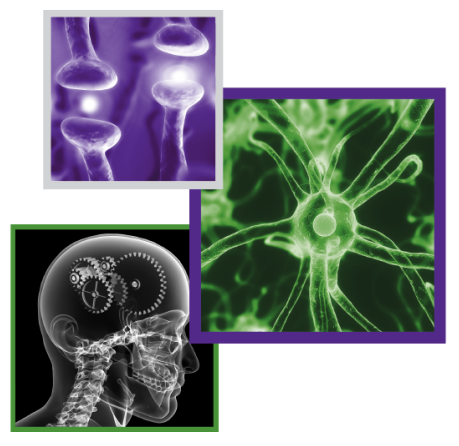

\author{
Amelia D Moser ${ }^{1}$, Eric Epping ${ }^{1}$, Patricia Espe-Pfeifer ${ }^{2}$, Erin Martin'1, Leah Zhorne ${ }^{3}$, \\ Katherine Mathews ${ }^{3}$, Martha Nance ${ }^{4}$, Denise Hudgell ${ }^{5}$, Oliver Quarrell ${ }^{6}$ \& Peg Nopoulos ${ }^{*}, 7$ \\ ${ }^{1}$ Department of Psychiatry, University of lowa Carver College of Medicine, lowa City, IA, USA \\ ${ }^{2}$ Departments of Psychiatry \& Pediatrics, University of lowa Carver College of Medicine, Iowa City, IA, USA \\ ${ }^{3}$ Departments of Pediatrics \& Neurology, University of lowa Carver College of Medicine, lowa City, IA, USA \\ ${ }^{4}$ Struthers Parkinson's Center, Minneapolis, MN, USA \\ ${ }^{5}$ Help4HD International, Santa Maria, CA, USA \\ ${ }^{6}$ Department of Clinical Genetics, Sheffield Children's Hospital, Sheffield, UK \\ ${ }^{7}$ Departments of Psychiatry, Pediatrics, \& Neurology, University of lowa Carver College of Medicine, lowa City, IA, USA \\ * Author for correspondence: Tel.: +1 319384 9264; Fax: +1 319353 8353; peggy-nopoulos@uiowa.edu
}

\section{Summary points}

- Juvenile onset Huntington's disease (JHD) is ultra-rare which results in gaps in knowledge of the phenomenology of symptoms.

- Adult onset HD (AOHD) and JHD have, in general, overlapping typical symptoms with motor, cognitive and psychiatric problems.

- Yet there are some unique features of JHD such as motor manifestation that are the opposite of those seen in AOHD (hyperkinesis in AOHD and hypokinesis in JHD).

- There may be additional symptoms that are common but unrecognized in JHD.

- Social media has provided a format in which families of children with rare diseases such as JHD can communicate on a regular basis regarding their children.

- A web-based survey was created assessing the phenomenology of a set of five symptoms felt by families to be common but under-recognized: pain, itching, sleeping difficulties, psychosis and tics.

- This survey was distributed to caretakers of JHD patients to evaluate the phenomenology of these symptoms.

- Disrupted sleep was the most prevalent symptom (87\%), followed by tics (78\%), pain (69\%), itching $(60 \%)$ and psychosis (39\%).

- There are symptoms in the JHD population that are not considered classic, however, are common and cause significant issues for both patients and caregivers.

Aim: The symptoms of Huntington's disease are well known, yet the symptoms of juvenile Huntington's disease (JHD) are less established due to its rarity. The study examined a cluster of symptoms considered to be common, but under-recognized in JHD: pain, itching, sleeping difficulties, psychosis and tics. Materials \& methods: A symptom survey was constructed using the online tool Qualtrics and dispersed to JHD caregivers through websites. Results: A total of 33 surveys were completed. Disrupted sleep was the most prevalent symptom (87\%), followed by tics $(78 \%)$, pain $(69 \%)$, itching $(60 \%)$ and psychosis $(39 \%)$. Conclusion: Despite limitations, the study supports that there are symptoms in the JHD population that are not considered classic, however, are common and significant for patients and caregivers.

First draft submitted: 09 May 2017; Accepted for publication: 13 July 2017; Published online: 18 October 2017

Keywords: Huntington's disease $\bullet$ itching $\bullet$ juvenile onset $\bullet$ pain $\bullet$ psychosis $\bullet$ sleep $\bullet$ symptoms $\bullet$ tics

Huntington's disease (HD) is a neurodegenerative disorder resulting from an expanded CAG trinucleotide repeat within the first exon of the HTT gene [1]. Typical symptoms of HD are motor, cognitive and psychiatric symptoms that progressively worsen. While patients are usually diagnosed in midlife (referred to here as adult onset HD or AOHD), when symptoms present early with a diagnosis by the age of 21 or younger, it is referred to as juvenile 
Huntington's disease (JHD). The length of the CAG repeat in HTT is directly related to age of onset with longer repeats (greater degree of mutation) being associated with earlier age of onset. Therefore, where AOHD subjects have an average range of CAG repeats around 45, most JHD subjects have CAG repeat lengths at 60 and above [1].

There is substantial overlap in the symptomatology of JHD and AOHD, including the fact that most cases begin with cognitive and/or behavioral issues that predate motor symptoms, sometimes by many years. However, there are distinct features of JHD, the most prominent being that the motor presentation is typically hypokinetic with stiffness and bradykinesia rather than the hyperkinesis of chorea that is classic for AOHD [2]. Seizures are another symptom unique to JHD [3].

In addition to the classic symptoms, many caregivers report a wide variety of additional symptoms that may or may not be related to the disease process. HD is rare, occurring in roughly 6-14 per 100,000 people [3]. Within cases of HD, roughly $5 \%$ are classified as JHD, making the incidence of JHD ultra-rare [4]. This is one of the main contributing factors to the ongoing gaps in knowledge about JHD, with the entirety of what we know being based on clinical experience, case reports and retrospective case series.

Recognizing symptoms that are not typical, yet directly relate to the disease process could have a major impact on awareness for the medical community with further attention to better assessment and potential treatments. However, while some symptoms may be seen as specific to JHD, they may be secondary to primary symptoms (e.g., pain could be secondary to dystonia and muscle stiffness). There are at least three ways in which symptoms can be evaluated to address whether they are disease-related: first, high prevalence. Although some symptoms may occur rarely in JHD and still be part of the disease process, if symptoms are seen commonly among patients, then support is given to the notion that the symptom is related to JHD. Second, the symptom is also seen in AOHD. There is substantial overlap in symptoms between AOHD and JHD, and if a symptom seen in JHD is also well-recognized in AOHD, then it suggests that it is a disease manifestation. At last, the relationship to the gene. A more direct assessment of whether or not a JHD symptom is related to disease process is to evaluate its relationship to CAG repeat length.

One of the advantages of the advent of social media is that groups of people from all over the world can communicate on a regular basis. The JHD community is an excellent example of platforms such as Facebook and Twitter providing ongoing communication between caregivers of children with rare diseases. This has led to discussions about symptoms that they saw frequently in their children, but because they were not classical, families questioned whether or not the symptom was related to JHD. This prompted our group to join with active members of the JHD community to devise a survey that was focused specifically on what might be considered as under-recognized but common symptoms of JHD: pain, sleep, itching, psychosis and tics. In addition, each symptom was evaluated for relationship to CAG length as a method to directly link the symptom with the disease process.

\section{Methods}

A survey was composed focusing on specific symptoms that were commonly discussed among caregivers of patients with JHD. See Box 1 for the complete survey. A total of five symptoms were included: pain, sleep disturbance, itching, psychosis (defined as hallucinations or delusions) and tics (defined as brief, repetitive, spasm-like movements or vocalizations such as grunts, coughs, or sniffs). Although psychosis and tics can be considered under the traditional headings of psychiatric or motor symptoms, respectively, their frequency in JHD is unknown.

If your child has had any additional symptoms that you would like to explain to us or if you would like to provide more information about your responses above, please describe here.

The survey was completed by the caregiver and asked a variety of questions covering demographic information, including the child's gender, current age, age at which they were diagnosed and CAG repeat length. For the purposes of this survey, JHD was defined by an age at diagnosis of 21 or younger and corroborated by a CAG count of at least 40 . While several other studies of JHD have utilized an age of onset rather than diagnosis, in a survey format there was concern that the more difficult to report age of onset would be less accurate [2,5]. The survey contained questions about symptoms their child may be experiencing. For each symptom, the survey inquired whether that symptom was present in the JHD subject. If they answered yes, then the respondents were asked additional information on the age of onset, severity, frequency and nature of each of the symptoms. Severity ratings were for the purpose of understanding how the caretaker and child experienced the symptoms, and the options were mild, moderate, or severe. As such, these were left up to the judgment of the caretaker rather than assigned specific meaning by the researchers. 
Box 1. The survey.

This survey is designed to evaluate symptoms of juvenile Huntington's disease (JHD) that may occur frequently but have been poorly recognized by healthcare professionals.

Please answer the following questions about your child or ward who has been diagnosed with JHD:

- Current age

- Gender

- Age at which they were diagnosed

- CAG repeat length

Have you witnessed any of the following symptoms affecting your child/ward?

- Pain

- Yes/no; if yes, answer the following:

- How old was your child/ward when it began?

- Rate the severity: mild/moderate/severe

- How often does pain occur? Is there a noticeable pattern?

- Describe the nature of the pain. For example, is it sharp, dull, intermittent, constant?

- Where is the pain located? Is it always in the same place?

- Have you discussed your child/ward's pain symptoms with medical providers? What treatment options did they suggest?

- Has there been treatment that has been helpful? If yes, please describe

- Disrupted sleep/insomnia (Sleep problems)

- Yes/no; if yes, answer the following:

- How old was your child/ward when it began?

- Rate the severity: mild/moderate/severe

- How often do sleep disturbances occur? Is there a noticeable pattern?

- Describe the nature of the insomnia. For example, is it inability to fall asleep, inability to stay asleep or both?

- Have you discussed your child/ward's sleep disturbances with medical providers? What treatment options did they suggest?

- Has there been treatment that has been helpful? If yes, please describe

- Itching

- Yes/no; if yes, answer the following:

- How old was your child/ward when it began?

- Rate the severity: mild/moderate/severe

- How often does itching occur? Is there a noticeable pattern?

- Describe the nature of the itching. For example, where is the itching located - is it always in the same place? Is there an associated rash or change in skin?

- Have you discussed your child/ward's itching with medical providers? What treatment options did they suggest?

- Has there been treatment that has been helpful? If yes, please describe

- Psychosis (hallucinations or ideas that are not based in reality)

- Yes/no; if yes, answer the following:

- How old was your child/ward when it began

- Rate the severity: mild/moderate/severe

- How often do psychotic episodes occur? Is there any noticeable pattern or instigating factor?

- Describe the nature of the psychosis. Does your child/ward experience visual hallucinations (seeing things that are not there) or auditory hallucinations (hearing things)? Does your child/ward express delusions (beliefs not based in reality)?

- Have you discussed your child/ward's psychosis with medical providers? What treatment options did they suggest?

- Has there been treatment that has been helpful? If yes, please describe

- Tics (repetitive movement that is not chorea; eye blinks, sniffs, head jerks)

- Yes/no; if yes, answer the following:

- How old was your child/ward when it began?

- Rate the severity: mild/moderate/severe

- How often do tics occur? Is there a noticeable pattern or instigating factor?

- Describe the nature of the tics. For example, where are they located? What kind of movement do they involve?

- Have you discussed your child/ward's tics with medical providers? What treatment options did they suggest?

- Has there been treatment that has been helpful? If yes, please describe 
Table 1. Symptom prevalence and severity.

\begin{tabular}{|llllll|}
\hline Symptom & $\%$ reported $(\mathbf{n}=\mathbf{3 3})$ & Number of subjects & Mild $(\%)$ & Moderate $(\%)$ & Severe $(\%)$ \\
\hline Sleeping & 87 & 25 & 12 & 40 & 32 \\
\hline Tics & 78 & 19 & 47 & 50 & 21 \\
\hline Pain & 69 & 18 & 28 & 41 & 22 \\
\hline Itching & 17 & 18 & 29 & 41 \\
\hline Psychosis & 60 & 7 & 57 & 14 \\
\hline
\end{tabular}

\section{Table 2. Symptom frequency.}

\begin{tabular}{|lllll|}
\hline Symptom & Number of subjects & Constantly (\%) & \multicolumn{2}{c}{ Daily/almost daily (\%) } \\
\hline Sleep & 22 & 0 & 68 & 31 \\
\hline Pain & 17 & 23 & 47 & 30 \\
\hline Itching & 12 & 50 & 33 & 33 \\
\hline Psychosis & 3 & 0 & 47 & 67 \\
\hline Tics & 15 & 20 & 33 \\
\hline
\end{tabular}

\begin{tabular}{|c|c|c|c|c|c|}
\hline Symptom & Number of subjects & Prediagnosis (\%) & $\begin{array}{l}\text { Mean length of time prior } \\
\text { to diagnosis (range) }\end{array}$ & Postdiagnosis (\%) & $\begin{array}{l}\text { Mean length of time } \\
\text { postdiagnosis (range) }\end{array}$ \\
\hline Pain & 20 & 35 & 2.5 years ( $1-6$ years) & 65 & 2.1 years ( $0-7$ years) \\
\hline Sleep & 25 & 48 & 7.2 years ( $0.5-21$ years) & 52 & 1.5 years ( $0-5$ years) \\
\hline Itching & 17 & 35 & 2.3 years ( $1-8$ years) & 65 & 2.1 years ( $0-7$ years) \\
\hline Psychosis & 7 & 43 & 2.0 years ( $1-3$ years) & 57 & 2.0 years ( $0-4$ years) \\
\hline Tics & 20 & 50 & 4.3 years ( $1-12$ years) & 50 & 1.8 years ( $0-6$ years) \\
\hline
\end{tabular}

The study was approved by the University of Iowa Institutional Review Board. The survey was constructed in Qualtrics and began with an informed consent page which was required to be completed before gaining access to the survey. The link to the survey was posted on a variety of HD and JHD Facebook and Twitter accounts, and was open for response for 16 weeks.

To evaluate the relationship of each symptom to CAG repeat length, $t$-tests (two-sided) were used to compare the mean CAG lengths of those with and without the symptom. In addition, a Spearman's rank-order correlation was run to determine if there was a relationship between symptom severity and CAG repeat length.

\section{Results}

A total of 42 surveys were completed. Several surveys were excluded because the age of diagnosis was later than 21 years $(n=4)$ or there was no CAG repeat included to confirm their diagnosis $(n=5)$. Of the remaining 33 JHD subjects, there were 18 males and 15 females. Mean age at diagnosis was 12.21 years (range 3-21 years), mean current age was 15.75 years (range 4-27 years) and mean CAG repeat length was 76.09 (range 45-109). Table 1 indicates the percentage of respondents that answered yes to whether or not a symptom was present, as well as the severity of each symptom (mild/moderate/severe). Of the five symptoms included, sleep difficulties were most common followed by tics, pain, itching and psychosis. Table 2 describes the frequency with which each symptom was present. Finally, Table 3 reports when each symptom developed within the course of the disease.

\section{Sleep disturbances}

Caretakers of patients with JHD indicated that sleep disruption was nearly ubiquitous. Not only common, this symptom was of major significance as of those who identified sleep disturbance, $88 \%$ indicated it was moderate to severe and over half indicated that it was a nightly or 'almost nightly' issue. Most of these complaints centered on falling asleep or both falling asleep and maintaining sleep. Approximately half of respondents indicated sleep disturbance preceded diagnosis by several years. This included five caretakers who indicated their child had suffered from disrupted sleep since birth. Of those who reported the symptom after diagnosis, the mean length of time after 
Table 4. Relationship between symptom and CAG repeat length.

\begin{tabular}{|llll}
\hline Symptoms & Yes & No & $t$-test (p) \\
\hline Sleep: CAG repeat length mean (SD) & $76.10(18.54)$ & $76.0(22.07)$ & $0.01(0.99)$ \\
\hline Pain: CAG repeat length mean (SD) & $79.43(17.51)$ & $68.40(19.68)$ & $1.60(0.11)$ \\
\hline Itching: CAG repeat length mean (SD) & $82.10(14.63)$ & $66.85(20.78)$ & $2.47(0.01)$ \\
\hline Tics: CAG repeat length mean (SD) & $74.65(18.00)$ & $81.43(21.34)$ & $0.85(0.40)$ \\
\hline Psychosis: CAG repeat length mean (SD) & $78.77(17.69)$ & $74.35(19.43)$ & $0.66(0.51)$ \\
\hline SD: Standard deviation. & & & \\
\hline
\end{tabular}

diagnosis was 1.5 years, suggesting that sleep disturbance is a problem occurring early in the course of disease. The mean age of sleep disturbance onset was 10.06 years, with a range of infancy to 23 years.

\section{Tics}

Just over three quarters of participants reported tics. Tics were rated as mild-to-moderate in most of the subjects, and over half complained of them daily or even constantly. All types of tics were reported but blinking and sniffing were most common, followed by tics of legs and arms. Like sleep, approximately half of respondents indicated that the tics occurred prior to diagnosis and $50 \%$ after diagnosis. The mean age of tic onset was 11.20 years with a range of 3-21 years.

\section{Pain}

Many respondents reported their child experienced some type of pain. Of those who indicated there was pain, almost three quarters rated the severity as moderate or severe. In terms of frequency, pain was rated most commonly as happening on a daily basis, with some indicating the pain was constant. The location of the pain went from head to toe, but legs were the most common complaint and were reported more than three-times as often as any other body part. A total of $73 \%$ respondents addressing the location of their pain reported leg pain. These pain symptoms tended to postdate diagnosis by an average of 2 years. The mean age of pain onset was 12.18 years, with a range of $3.5-22$ years.

\section{Itching}

Over half of respondents reported problems related to itching, with most complaining of moderate-to-severe ratings. The frequency of itching was high, with half of the respondents indicating it was a constant issue and an additional third reported itching occurred on a daily basis. Importantly, most respondents (80\%) reported no obvious skin irritation (prior to the consequences of the scratching itself), suggesting that the sensation of itching was not originating from a rash, but instead a sensory aberration that felt like something that scratching would relieve. Similar to the reports of pain, legs were a common site of itching $(40 \%)$, tied with full-body itching in frequency. Several complained of the abdomen and back itching as well. Complaints of itching more often occurred after diagnosis by approximately 2 years. The mean age of itching onset was 12.00 years with a range of 4-23 years.

\section{Psychosis}

This was defined in the survey as hallucinations or ideas that are not based on reality. Over a third of the participants reported observing psychosis in their children. There was a wide range of severity, with a majority choosing a rating of mild. The most common manifestations of these episodes were visual hallucinations, followed by auditory hallucinations. The number of subjects with psychosis was small $(n=7)$ with three noting symptoms prior to diagnosis and four noting symptoms after diagnosis. The mean age of psychosis onset was 9.43 years with a range of $4-15$ years.

\section{Relationship to CAG repeat length}

Age of diagnosis of JHD was highly correlated to CAG repeat length (Pearson $r=-0.79 ; p<0.001$ ). Table 4 and Figure 1 show the results of the group comparison evaluating mean CAG repeat length between those who have the symptoms and those who do not. The only symptom with a significant group difference was itching in which those with itching had a substantially longer CAG repeat length (mean $=82.10$; standard deviation $[\mathrm{SD}]=14.63$ ) compared with those without itching (mean $=66.85 ; \mathrm{SD}=20.78$ ). Further, it was shown that the severity of the 


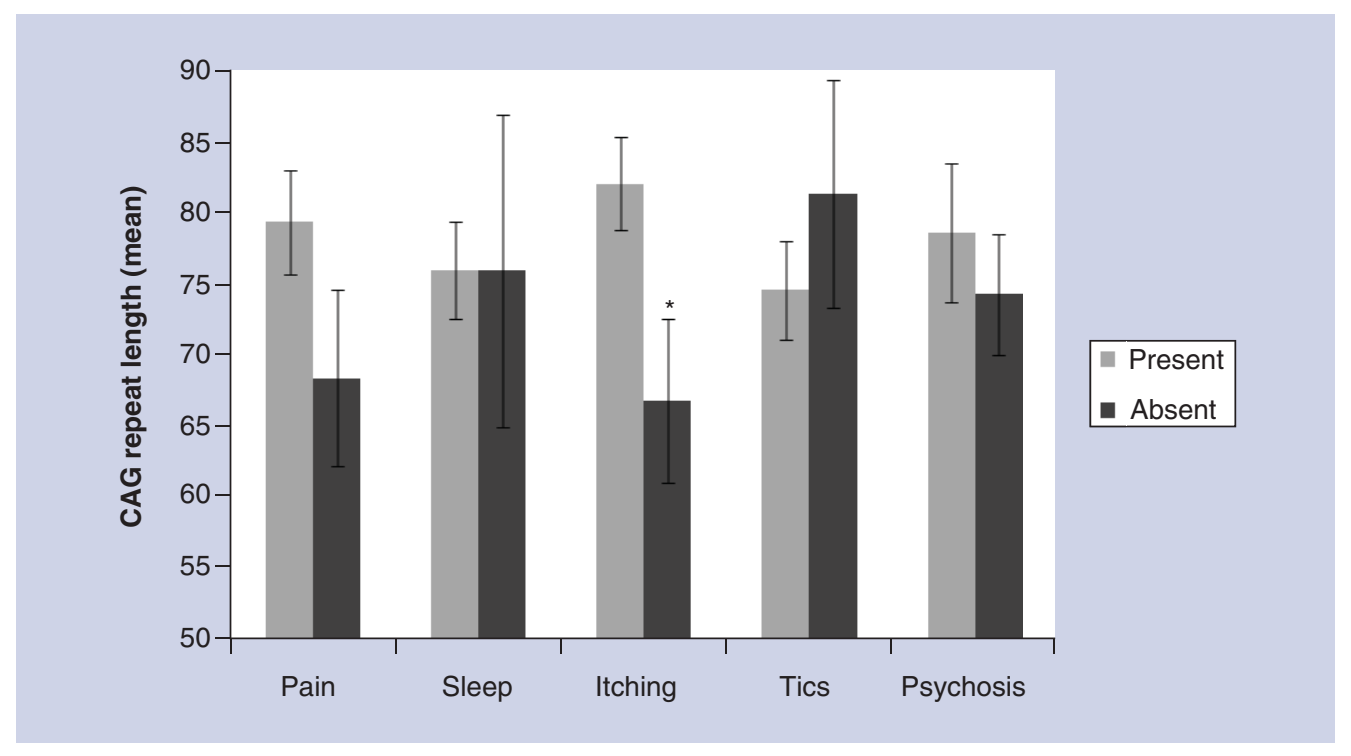

Figure 1. Evaluation of relationship between CAG repeat length and presence or absence of each of the five symptoms.

${ }^{*} \mathrm{p}=0.01$.

For each symptom, patients are divided into 'present' or 'absent' groups and mean CAG repeat length of each group is compared. Error bars are standard error. Subjects with itching had a substantially longer CAG repeat mean than those without the symptom.

itching symptom was also directly correlated with CAG repeat length, with higher repeat lengths associated with greater symptom severity $\left(\mathrm{r}_{\mathrm{s}}=0.56 ; \mathrm{p}=0.02\right)$.

\section{Discussion}

The findings from this survey identify symptoms that are not considered classic for JHD yet are quite common and most importantly, identified by caregivers as being some of the most difficult issues to deal with for their children.

The most common symptom identified by the survey was sleep disturbance. This is not entirely surprising, as other studies have found difficulties related to sleep in AOHD as well [6]. AOHD patients experience a range of sleeping problems, including trouble initiating and maintaining sleep, issues largely corresponding to complaints identified by caregivers of JHD patients.

Other symptoms related to AOHD or JHD, such as chorea, itching, or pain can contribute to sleep difficulties, but may not be the primary cause. When evaluated specifically, chorea was not the main instigator of sleep disruption in AOHD [7]. In addition, the current survey showed that sleep disturbance was commonly an early feature, predating diagnosis sometimes by many years. The true etiology of these sleep disturbances continues to be studied, and the current leading explanation is centered on abnormalities of the hypothalamus disrupting circadian rhythms [8].

Pain, most typically in the legs, was also very prominent among children with JHD. The fact that this was reported to be mostly moderate to severe and daily is a striking testament to the suffering of these patients. This is corroborated by a prior study based on interviews with caregivers for individuals with JHD which also identified pain as a significant concern [9]. The etiology of this pain is still unclear. It could potentially be due to dystonia or parkinsonism, however, with no correlation between pain and motor symptoms, it is difficult to support. Pain is a symptom not commonly reported in AOHD. There are very few studies of pain prevalence and a recent report found only three of $45 \mathrm{HD}$ patients complaining of chronic pain [10]. Additionally, findings have supported impaired processing of pain among those with $\mathrm{AOHD}$, suggesting that an increased threshold for pain may be present in AOHD, leading to a low incidence of chronic pain [10,11]. Further study is needed to better characterize this symptom in JHD.

Although the term itching is typically a symptom that refers to a dermatologic cause, JHD children suffer from some type of sensory phenomenon that mimics this sensation, as most of the respondents indicated there was no clear dermatologic change associated with the itching. This sensory phenomenon may potentially have properties 
of neuropathic itching, which is itself poorly understood but has been associated with CNS lesions affecting sensory pathways from stroke, or multiple sclerosis [12]. This phenomenon has not been studied in AOHD to our knowledge, and it is the least understood of the symptoms queried here. The fact that most patients with JHD experience this symptom after diagnosis suggests that it may be secondary, yet there is no clear feature of JHD that this symptom would be due to. The most important aspect of the itching symptom is that it was the only symptom investigated that had a direct relationship with CAG in which CAG repeat length was substantially longer in those who had itching compared with those that did not, and the greater the severity of the itching, the longer the CAG repeat length. Given the high association between length of CAG repeats and age of diagnosis, this effectively means that those with JHD who are diagnosed at the earliest ages are more likely to develop itching compared with those who develop it later. This is exactly the same pattern seen in seizures with JHD in which those with seizures have an earlier age of onset and greater CAG repeat length than those who do not [3]. Interestingly, seizures are a unique JHD symptom, and it appears that itching may be as well. Also, given its relationship to CAG repeat, it seems more likely to be a primary symptom rather than secondary.

Psychosis and tics are symptoms that are considered part of the traditional symptoms of JHD, being psychiatric and motor, respectively. Psychosis is indeed seen in AOHD, cited to have a prevalence of 3-11\% [13]. However in the current study, visual hallucinations were the most common feature of psychosis whereas in AOHD, delusions are most prominent [14]. Also, psychosis in AOHD has been reported to have a familial predisposition with familial aggregation, suggesting that other genetic factors may influence susceptibility to psychosis precipitated by CAG expansion in the HD gene [15].

The symptoms of tics may well be harder to distinguish from more typical motor problems such as chorea. Therefore, some of the movements interpreted as tics may instead be representative of other involuntary movements. However, we attempted to address this in the survey by indicating that we wanted respondents to rate 'repetitive movement that is not chorea; eye blinks, sniffs, head jerks'. These families are likely to know what chorea is as they are exposed to other relatives with AOHD. Tics have been previously reported in JHD case series [16], individual case reports [17] and even in nonhuman primate models of $\mathrm{HD}$ [18]. Few studies have documented the prevalence of tics in AOHD. One recent study found only two of 115 AOHD patients $(n=115)$ had tics [16]. The timing of tics in our study suggested that it is often present prior to diagnosis. This has been reported in the literature with case reports [19] and case series [20] documenting the presence of tics (blepharospasm in particular) prior to diagnosis in JHD. The pathology of JHD is occurring in the context of brain development and motor/vocal tics are a well-known developmental phenomenon that occurs in childhood with transient motor tics estimated to affect approximately $20 \%$ of children [20]. However, when severe or sustained, tics may represent Tourette syndrome, which is well-established to be due, in part, to striatal pathology, the same region of pathology as JHD [19] supporting the notion that tics are a primary manifestation of the disease process.

There are several limitations that may have impacted our study: first, the definition of JHD as diagnosed before 21 years old may under-represent individuals whose symptoms began in their late teen years but were diagnosed after the age of 21, as diagnosis may be delayed [21]. Yet in order to define our sample, we had to operationalize a definition of whom to include. Second, prevalence of symptoms in the JHD population was not compared with normative data. For instance, sleep disturbance has been quantified as $12 \%$ of school-aged children (aged 11-15 years) [22], and 37\% of 4-6 year olds have been reported to have recurrent leg pain often described as 'growing pains' [23]. Caregivers may also have had difficulty recognizing some of the symptoms, particularly in distinguishing tics from other motoric abnormalities, or pain from nondermatologic itching. There are also limitations specific to the use of surveys. A survey-based study does not include direct examination of the patient, which may have aided in confirmation of the JHD diagnosis and symptomatology. A survey also inherently relies upon self-selection for participation, and the group that chose to complete the survey may over-represent the frequency of symptoms. At last, a small sample size of 33 individuals may be inadequate to represent the broader population suffering from the disease. Nevertheless, in the context of an ultra-rare disease, this sample size is comparably large. Finally, the decision to focus on these specific symptoms was based informally from discussions within the JHD community and patients seen at the University of Iowa Huntington's Disease Center of Excellence. Other symptoms may also be common but not recognized.

This survey demonstrates that the symptoms we asked about are quite common and often severe. This provides a focal point for further exploration. Simply providing awareness of these symptoms to the medical community can help to improve clinical assessments by inquiring about these symptoms as many caregivers may also tend to discount certain symptoms such as itching to another etiology rather than JHD. More importantly, more work 
is needed to better understand the pathophysiology underlying the symptoms, and to develop more effective treatments to improve the quality of life for patients with JHD. Researchers should move forward with studies that include direct examination of JHD patients and questionnaires that more precisely examine aspects of their classical and newly-recognized symptoms. Finally, these same symptoms should be explored within the AOHD community to better understand whether these are unique to those with juvenile onset.

\section{Author contributions}

The conception, organization and execution of the research project were carried out by D Hudgell and P Nopoulos. The design and execution of statistical analysis were done by P Nopoulos. Review and critique were done by A Moser, E Epping, P Espe-Pfeifer, E Martin, L Zhorne, K Mathews, M Nance, D Hudgell, O Quarrell and P Nopoulos. In terms of manuscript preparation: writing of the first draft was done by A Moser and P Nopoulos and; the review and critique were done by A Moser, E Epping, P Espe-Pfeifer, E Martin, L Zhorne, K Mathews, M Nance, D Hudgell, O Quarrell and P Nopoulos.

Financial \& competing interests disclosure

E Epping: Growth and Development of the Striatum in Huntington's Disease (4R01NS055903-08). K Mathews: Muscular Dystrophy Cooperative Research Center; Project II, NINDS (U54 NS05367212). P Nopoulos: Growth and Development of the Striatum in Huntington's Disease (4R01NS055903-08); Longitudinal Assessment of Brain Structure and Function in Myotonic Dystrophy (5R01NS094387-02); Preterm Transfusions: Brain Structure and Function Outcomes (4P01HL046925-20). The authors have no other relevant affiliations or financial involvement with any organization or entity with a financial interest in or financial conflict with the subject matter or materials discussed in the manuscript apart from those disclosed.

No writing assistance was utilized in the production of this manuscript.

Ethical conduct of research

This study was approved by the University of lowa Institutional Review Board. The authors confirm that they have read the Journal's position on issues involved in ethical publication and affirm that this work is consistent with those guidelines.

\section{Open access}

This work is licensed under the Attribution-NonCommercial-NoDerivatives 4.0 Unported License. To view a copy of this license, visit http://creativecommons.org/licenses/by-nc-nd/4.0/

\section{References}

Papers of special note have been highlighted as: • of interest; $\bullet \bullet$ of considerable interest

1. Barker R, Squitieri F. The clinical phenotype of juvenile Huntington's Disease. In: Juvenile Huntington's Disease: and Other Trinucleotide Repeat Disorders. Quarrell OWJ, Brewer HM, Squitieri F, Barker RA, Nance MA, Landwehrmeyer GB (Eds). Oxford University Press, NY, USA (2009).

-. Offers a comprehensive view of juvenile Huntington's disease (JHD) through in-depth discussions of research as well as contributions from families in the population.

2. Quarrell OW, Nance MA, Nopoulos P, Paulsen JS, Smith JA, Squitieri F. Managing juvenile Huntington's disease. Neurodegener. Dis. Manag. 3(3), 267-276 (2013).

3. Baig SS, Strong M, Quarrell OW. The global prevalence of Huntington's disease: a systematic review and discussion. Neurodegener. Dis. Manag. 6(4), 331-343 (2016).

4. Quarrell O, O’Donovan KL, Bandmann O, Strong M. The prevalence of juvenile Huntington's disease: a review of the literature and meta-analysis. PLoS Curr. 4, e4f8606b742ef3 (2012).

5. Nance MA, Myers RH. Juvenile onset Huntington's disease: clinical and research perspectives. Ment. Retard. Dev. Disabil. Res. Rev. 7(3), 153-157 (2001).

- Details research findings and the clinical phenotype associated with JHD.

6. Goodman AO, Morton AJ, Barker RA. Identifying sleep disturbances in Huntington's disease using a simple disease-focused questionnaire. PLoS Curr. 2, RRN1189 (2010).

7. Fish DR, Sawyers D, Allen PJ, Blackie JD, Lees AJ, Marsden CD. The effect of sleep on the dyskinetic movements of Parkinson's disease, Gilles de la Tourette syndrome, Huntington's disease and torsion dystonia. Arch. Neurol. 48(2), 210-214 (1991).

8. Morton AJ. Circadian and sleep disorder in Huntington's disease. Exp. Neurol. 243, 34-44 (2013).

9. Smith JA, Brewer HM, Eatough V, Stanley CA, Glendinning NW, Quarrell OW. The personal experience of juvenile Huntington's disease: an interpretative phenomenological analysis of parents' accounts of the primary features of a rare genetic condition. Clin. Genet. 69(6), 486-496 (2006). 
-• One of only a few that thoroughly examines the broader impact JHD has on the family, and the progression of the disease through their eyes.

10. de Tommaso M, Franco G, Ricci K, Montemurno A, Sciruicchio V. Laser evoked potentials in early and presymptomatic Huntington's disease. Behav. Neurol. 2016, 8613729 (2016).

11. Perrotta A, Serpino C, Cormio C et al. Abnormal spinal cord pain processing in Huntington's disease. The role of the diffuse noxious inhibitory control. Clin. Neurophysiol. 123(8), 1624-1630 (2012).

12. Oaklander AL. Neuropathic itch. Semin. Cutan. Med. Surg. 30(2), 87-92 (2011).

13. van Duijn E, Kingma EM, van der Mast RC. Psychopathology in verified Huntington's disease gene carriers. J. Neuropsychiatry Clin. Neurosci. 19(4), 441-448 (2007).

14. Paulsen JS, Ready RE, Hamilton JM, Mega MS, Cummings JL. Neuropsychiatric aspects of Huntington's disease. J. Neurol. Neurosurg. Psych. 71(3), 310-314 (2001).

15. Tsuang D, Almqvist EW, Lipe $\mathrm{H}$ et al. Familial aggregation of psychotic symptoms in Huntington's disease. Am. J. Psych. 157(12), 1955-1959 (2000).

16. Liu ZJ, Sun YM, Ni W, Dong Y, Shi SS, Wu ZY. Clinical features of Chinese patients with Huntington's disease carrying CAG repeats beyond 60 within HTT gene. Clin. Genet. 85(2), 189-193 (2014).

17. Xing S, Chen L, Chen X, Pei Z, Zeng J, Li J. Excessive blinking as an initial manifestation of juvenile Huntington's disease. Neurol. Sci. 29(4), 275-277 (2008).

18. Palfi S, Brouillet E, Jarraya B et al. Expression of mutated huntingtin fragment in the putamen is sufficient to produce abnormal movement in nonhuman primates. Mol. Ther. 15(8), 1444-1451 (2007).

19. Pappas SS, Leventhal DK, Albin RL, Dauer WT. Mouse models of neurodevelopmental disease of the basal ganglia and associated circuits. Curr. Top. Dev. Biol. 109, 97-169 (2014).

20. Scahill L, Specht M, Page C. The prevalence of tic disorders and clinical characteristics in children. J. Obsessive Compuls. Relat. Disord. 3(4), 394-400 (2014).

21. Ribai P, Nguyen K, Hahn-Barma V et al. Psychiatric and cognitive difficulties as indicators of juvenile Huntington's disease onset in 29 patients. Arch. Neurol. 64(6), 813-819 (2007).

- Examines a large sample of 29 patients with JHD and reports their symptomatology and diagnosis process.

22. Fricke-Oerkermann L, Pluck J, Schredl M et al. Prevalence and course of sleep problems in childhood. Sleep 30(10), 1371-1377 (2007).

23. Evans AM, Scutter SD. Prevalence of 'growing pains' in young children. J. Pediatr. 145(2), 255-258 (2004). 